PROCEEDINGS OF THE

AMERICAN MATHEMATICAL SOCIETY

Volume 140, Number 7, July 2012, Pages 2527-2535

S 0002-9939(2012)11128-1

Article electronically published on January 18, 2012

\title{
ON THE CONVEX HULL OF SYMMETRIC STABLE PROCESSES
}

\author{
JÜRGEN KAMPF, GÜNTER LAST, AND ILYA MOLCHANOV
}

(Communicated by Richard C. Bradley)

\begin{abstract}
Let $\alpha \in(1,2]$ and $X$ be an $\mathbb{R}^{d}$-valued symmetric $\alpha$-stable Lévy process starting at 0 . We consider the closure $S_{t}$ of the path described by $X$ on the interval $[0, t]$ and its convex hull $Z_{t}$. The first result of this paper provides a formula for certain mean mixed volumes of $Z_{t}$ and in particular for the expected first intrinsic volume of $Z_{t}$. The second result deals with the asymptotics of the expected volume of the stable sausage $Z_{t}+B$ (where $B$ is an arbitrary convex body with interior points) as $t \rightarrow 0$. For this we assume that $X$ has independent components.
\end{abstract}

\section{INTRODUCTION AND MAIN RESUlTS}

For fixed $\alpha \in(1,2]$ and fixed integer $d \geq 1$ we consider an $\mathbb{R}^{d}$-valued symmetric $\alpha$-stable Lévy process $X \equiv(X(t))_{t>0}$ starting at the origin. If $\alpha=2$ and the components of $X$ are independent with variance 1, then $X$ is a standard Brownian motion.

Let $Z$ be the closure of the convex hull of $\{X(t): 0 \leq t \leq 1\}$. A classical result of [16] for planar standard Brownian motion says that

$$
\mathbb{E} V_{1}(Z)=\sqrt{2 \pi}
$$

where $V_{1}(Z)$ denotes half the perimeter of $Z$. Our first aim in this paper is to formulate and to prove such a result for arbitrary $\alpha \in(1,2]$ and arbitrary dimension $d$. In fact we also consider more general geometric functionals; see Theorem 1.1. To the best of our knowledge our paper is the first dealing with the convex hull $Z$ in the case $\alpha \in(1,2)$.

Let $B \subset \mathbb{R}^{d}$ be a convex body, that is a non-empty compact and convex subset of $\mathbb{R}^{d}$. Then the (random) set $\bigcup_{s \leq t}(X(s)+B)$ is called stable sausage up to time $t$. The asymptotic behaviour of the volume of this sausage as $t \rightarrow \infty$ has been studied intensively; cf. [15] for the case of Brownian motion and [5, 11] for the case of more general stable processes. The second aim of our paper is to start an investigation of the asymptotic behaviour as $t \rightarrow 0$; see Theorem [1.7. For this, we assume that the process $X$ has independent components. In the case $\alpha=2$ the stable sausage is known as Wiener sausage. Even then our Theorem 1.7 constitutes a new result; see Corollary 1.8

Received by the editors December 9, 2010 and, in revised form, February 25, 2011

2010 Mathematics Subject Classification. Primary 60G52; Secondary 28A75, 60D05.

The third author was partially supported by Swiss National Science Foundation Grant No. 200021-126503.

The authors are grateful to the referee for a careful reading of the paper.

(C)2012 American Mathematical Society Reverts to public domain 28 years from publication 2527 
By definition, the process $X$ has stationary independent increments, and the distribution of $X(t)$ is symmetric $\alpha$-stable for each $t \geq 0$. By [12, Theorem 2.4.3] there is a symmetric finite measure $\Gamma$ (unique in case $\alpha<2$ ) on the unit sphere $S^{d-1}$ in $\mathbb{R}^{d}$ such that

$$
\mathbb{E} \exp [i\langle u, X(t)\rangle]=\exp \left[-t^{\alpha} \int|\langle u, v\rangle|^{\alpha} \Gamma(d v)\right], \quad u \in \mathbb{R}^{d}, t \geq 0,
$$

where $\langle\cdot, \cdot\rangle$ denotes the Euclidean scalar product. The measure $\Gamma$ is the spectral measure of $X(1)$. The Minkowski inequality implies that $\left(\int|\langle u, v\rangle|^{\alpha} \Gamma(d v)\right)^{1 / \alpha}$ is a sublinear function of $u$. Moreover, this function is also positively homogeneous. Theorem 1.7.1 in [13] implies that there is a convex body $K$ (uniquely determined by $\Gamma$ ) such that

$$
\mathbb{E} \exp [i\langle u, X(t)\rangle]=\exp \left[-t h(K, u)^{\alpha}\right], \quad u \in \mathbb{R}^{d}, t \geq 0,
$$

where

$$
h(K, u):=\sup \{\langle u, x\rangle: x \in K\}, \quad u \in \mathbb{R}^{d},
$$

is the support function of $K$. We remark that $K$ is also determined uniquely by the distribution of $X(1)$ in the case $\alpha=2$. The body $K$ is symmetric and contains the origin 0 . However, 0 does not need to be an interior point of $K$. The set $K$ is called the associated zonoid of $X(1)$; see [10]. Zonoids form a subfamily of convex bodies that are obtained as limits (with respect to the Hausdorff distance) of Minkowski (or elementwise) sums of segments, where the Minkowski sum is defined as $B+C:=\{x+y: x \in B, y \in C\}$ for two sets $B, C \subset \mathbb{R}^{d}$. The set $K$ from (1.3) belongs to the family of $L_{p}$-zonoids with $p=\alpha$, which appear as limits for $L_{p}$-sums (or Firey sums) of segments; see [4, 10].

As in [10] it is useful to introduce the polar set

$$
F:=\left\{u \in \mathbb{R}^{d}: h(K, u) \leq 1\right\}
$$

of the associated zonoid $K$. This set is convex, closed and origin-symmetric and satisfies $\|u\|_{F}=h(K, u)$ for all $u \in \mathbb{R}^{d}$, where

$$
\|u\|_{F}:=\inf \{s \geq 0: u \in s F\}
$$

is the Minkowski functional of $F$; see [13, Sec. 1.6,1.7] for more details in case 0 is an interior point of $K$. Therefore,

$$
\mathbb{E} \exp [i\langle u, X(t)\rangle]=\exp \left[-t\|u\|_{F}^{\alpha}\right], \quad u \in \mathbb{R}^{d}, t \geq 0 .
$$

If $\alpha=2$, then $X$ is a Gaussian process and both $F$ and $K$ are ellipsoids. In particular, if $F$ (and also $K$ ) is a Euclidean ball, then the components of $X$ are independent Brownian motions, which become standard if the radius of $F$ is $\sqrt{2}$.

Note that the process $X$ is self-similar in the sense that $(X(s t))_{s \geq 0} \stackrel{d}{=} t^{1 / \alpha} X$ for any $t>0$; see [12, Ex. 7.1.3] and [6, Ch. 15]. For $t \geq 0$, let $S_{t}$ be the closure of the path $S_{t}^{0}:=\{X(s): 0 \leq s \leq t\}$ and let $Z_{t}$ denote the convex hull of $S_{t}$. In Lemma 2.1 we will show that these are random closed sets; see [9] or [14] for the notion of a random closed set. We abbreviate $Z:=Z_{1}$. By self-similarity

$$
Z_{t} \stackrel{d}{=} t^{1 / \alpha} Z, \quad t>0
$$

In this paper we study geometric functionals of the random convex set $Z$, namely intrinsic and mixed volumes. The intrinsic volumes $V_{j}(K), j=0, \ldots, d$, of a convex 
body $K$ are the unique real numbers satisfying the Steiner formula

$$
V_{d}\left(K+t B^{d}\right)=\sum_{j=0}^{d} \kappa_{d-j} t^{d-j} V_{j}(K), \quad t \geq 0,
$$

where $B^{d}$ is the closed Euclidean unit ball in $\mathbb{R}^{d}$ and $\kappa_{j}$ is the $j$-dimensional volume of $B^{j}$. In particular, $V_{d}(K)$ is the volume of $K$ (justifying the use of the same symbol $V_{d}$ for the Lebesgue measure), $V_{d-1}(K)$ is half the surface area, $V_{d-2}(K)$ is proportional to the integrated mean curvature, $V_{1}(K)$ is proportional to the mean width of $K$, and $V_{0}(K)=1$. (If $d=2$, then the first intrinsic volume $V_{1}$ appears in (1.1).) The mixed volumes $V(K[j], L[d-j]), j=0, \ldots, d$, of two convex bodies $K, L \subset \mathbb{R}^{d}$ are the unique real numbers such that

$$
V_{d}(K+t L)=\sum_{j=0}^{d}\left(\begin{array}{l}
d \\
j
\end{array}\right) t^{d-j} V(K[j], L[d-j]), \quad t \geq 0 .
$$

Taking $L:=B^{d}$ in (1.6) and comparing with (1.5) shows that the intrinsic volumes are special mixed volumes:

$$
V_{j}(K)=\frac{\left(\begin{array}{l}
d \\
j
\end{array}\right)}{\kappa_{d-j}} V\left(K[j], B^{d}[d-j]\right), \quad j=0, \ldots, d .
$$

A geometric interpretation of the mixed volume $V(K[d-1], L[1])$ can be derived from (1.6):

$$
V(K[d-1], L[1])=\lim _{t \downarrow 0} t^{-1}\left(V_{d}(K+t L)-V_{d}(K)\right) .
$$

For more information on intrinsic and mixed volumes the reader is referred to [13].

Theorem 1.1. Let $B$ be a convex body in $\mathbb{R}^{d}$. Then

$$
\mathbb{E} V(B[d-1], Z[1])=\frac{\alpha}{\pi} \Gamma\left(1-\frac{1}{\alpha}\right) V(B[d-1], K[1]),
$$

where $\Gamma$ is the Gamma-function and $K$ is the associated zonoid of X(1). In particular,

$$
\mathbb{E} V_{1}(Z)=\frac{\alpha}{\pi} \Gamma\left(1-\frac{1}{\alpha}\right) V_{1}(K) .
$$

Remark 1.2. By the scaling relation (1.4) and the homogeneity property of mixed volumes [13, Eq. (5.1.24)], the identity (1.8) can be generalized to

$$
\mathbb{E} V\left(B[d-1], Z_{t}[1]\right)=\frac{\alpha}{\pi} \Gamma\left(1-\frac{1}{\alpha}\right) t^{1 / \alpha} V(B[d-1], K[1]) .
$$

A similar remark applies to all results of this paper.

Remark 1.3. The mixed volumes $V\left(K_{1}, \ldots, K_{d}\right)$ of $d$ convex bodies $K_{1}, \ldots, K_{d}$ are defined as coefficients in the linear expansion of the volume of $\lambda_{1} K_{1}+\cdots+\lambda_{d} K_{d}$; see [13, Sec. 5.1]. Since these volumes are linear in each argument, Theorem 1.1 and all further results of this paper hold also for $V\left(K_{1}, \ldots, K_{d-1}, Z\right)$ with arbitrary convex bodies $K_{1}, \ldots, K_{d-1}$, so that (1.8) is recovered if $K_{1}=\cdots=K_{d-1}=B$.

The proof of Theorem 1.1 relies on the fact that

$$
\mathbb{E} h(Z, u)=\frac{\alpha}{\pi} \Gamma\left(1-\frac{1}{\alpha}\right) h(K, u),
$$


for all $u$ from the unit sphere $S^{d-1}$ in $\mathbb{R}^{d}$. Therefore the rescaled $K$ is the mean body of $Z$ [14, p. 146] or the selection expectation of $Z$ [9, Thm. 2.1.22].

It is known [10, Ex. 3.2] that $X(1)$ has i.i.d. components if and only if $F=\sigma B_{\alpha}^{d}$, where $\sigma>0$ is a scaling parameter and

$$
B_{\alpha}^{d}:=\left\{x \in \mathbb{R}^{d}:\left(\left|x_{1}\right|^{\alpha}+\cdots+\left|x_{d}\right|^{\alpha}\right) \leq 1\right\}
$$

is the $\ell_{\alpha}$-unit ball in $\mathbb{R}^{d}$. In this case the polar set $K$ to $F$ is $\sigma^{-1} B_{\alpha^{\prime}}^{d}$, where $B_{\alpha^{\prime}}^{d}$ is the $\ell_{\alpha^{\prime}}$-ball in $\mathbb{R}^{d}$ with $1 / \alpha+1 / \alpha^{\prime}=1$. For $\alpha=2$ and $K$ being the Euclidean ball of radius $1 / \sqrt{2}$, the following corollary provides a direct generalization of (1.1).

Corollary 1.4. If $X$ is a standard Brownian motion in $\mathbb{R}^{d}$, then

$$
\mathbb{E} V_{1}(Z)=\frac{2 \sqrt{2} \Gamma\left(\frac{d+1}{2}\right)}{\Gamma\left(\frac{d}{2}\right)} .
$$

A stable random vector is called subgaussian if it appears as a product of a Gaussian random vector and a power of an independent positive stable random variable; see [12]. It is noted in [10] that subgaussian stable distributions are characterised by the fact that their associated zonoid $K$ is an ellipsoid. In particular, if $F=K=B^{d}$, then the process $X(t)$ has identically distributed (but not independent for $\alpha \neq 2$ ) components and

$$
\mathbb{E} V_{1}(Z)=\frac{d \alpha}{\pi} \Gamma\left(1-\frac{1}{\alpha}\right) \frac{\kappa_{d}}{\kappa_{d-1}}=\frac{2 \alpha}{\sqrt{\pi}} \Gamma\left(1-\frac{1}{\alpha}\right) \frac{\Gamma\left(\frac{d+1}{2}\right)}{\Gamma\left(\frac{d}{2}\right)} .
$$

The general subgaussian case is handled in the following lemma by reducing it to a formula involving the surface area of an ellipsoid.

Lemma 1.5. Let $K$ be an ellipsoid in $\mathbb{R}^{d}$ with semi-axes $c_{1}, \ldots, c_{d}$. Then

$$
V_{1}(K)=\frac{2 c_{1} \cdots c_{d}}{d \kappa_{d-1}} V_{d-1}(F),
$$

where $V_{d-1}(F)$ is the half surface area of the polar ellipsoid $F$ to $K$.

Expressions for $V_{d-1}(F)$ by means of elliptical integral are given in [2] and [17].

In the case of Brownian motion it is possible to calculate the expectation of the second intrinsic volume $V_{2}(Z)$ of $Z$.

Proposition 1.6. Assume that $X$ is a standard Brownian motion in $\mathbb{R}^{d}$. Then

$$
\mathbb{E} V_{2}(Z)=(d-1) \frac{\pi}{2}
$$

Now consider a fixed convex body $B$ with non-empty interior and turn to the asymptotic behaviour of the volume of the stable sausage $S_{t}+B$ as $t \rightarrow 0$. For this, we assume that the process $X$ has independent components, i.e. the associated zonoid of $X(1)$,

$$
K=\left\{\left(c_{1} x_{1}, \ldots, c_{d} x_{d}\right):\left(x_{1}, \ldots, x_{d}\right) \in B_{\alpha^{\prime}}^{d}\right\},
$$

is a scaled $\ell_{\alpha^{\prime}}$-ball $B_{\alpha^{\prime}}^{d}$; see (1.9).

Theorem 1.7. Let $B$ be a convex body with non-empty interior. If $X$ is a symmetric $\alpha$-stable Lévy process with independent components and the associated zonoid $K$ is given by (1.10), then

$$
\lim _{t \rightarrow 0} t^{-1 / \alpha}\left(\mathbb{E} V_{d}\left(S_{t}+B\right)-V_{d}(B)\right)=\frac{d \alpha}{\pi} \Gamma\left(1-\frac{1}{\alpha}\right) V(B[d-1], K[1]) .
$$


Theorem 1.7 also holds for processes obtained as a linear transformation of a symmetric $\alpha$-stable Lévy process with independent components. Even in the case of a Wiener sausage, Theorem 1.7 yields a new result.

Corollary 1.8. Assume that $X$ is a Brownian motion and let $B$ be a convex body with non-empty interior. Then

$$
\lim _{t \rightarrow 0} t^{-1 / 2}\left(\mathbb{E} V_{d}\left(S_{t}+B\right)-V_{d}(B)\right)=\frac{d \sqrt{2}}{\sqrt{\pi}} V\left(B[d-1], B^{d}[1]\right) .
$$

If $B=B^{d}$, the limit is $2 \sqrt{2} \pi^{(d-1) / 2} / \Gamma(d / 2)$.

Remark 1.9. In the special case $d=3$ and $\alpha=2$ the classical result from [15] yields that

$$
\mathbb{E} V_{3}\left(S_{t}+r B^{3}\right)=\frac{4}{3} \pi r^{3}+4 \sqrt{2 \pi} r^{2} \sqrt{t}+2 \pi r t
$$

for any $r, t \geq 0$. The constant term in $t$ can be interpreted geometrically as $V_{3}\left(r B^{3}\right)$. A comparison with Corollary 1.8 yields that the coefficient of $\sqrt{t}$ can be interpreted as

$$
4 \sqrt{2 \pi} r^{2}=3 \mathbb{E} V\left(r B^{3}[2], Z[1]\right)=r^{2} \kappa_{2} \mathbb{E} V_{1}(Z),
$$

where we have used (1.7) and the homogeneity property of mixed volumes; see e.g. [13, Eq. (5.1.24)].

\section{Proofs}

We need the following measurability property of the closure $S_{t}$ of $\{X(s): 0 \leq$ $s \leq t\}$ and its convex hull $Z_{t}$, referring to 9 , 14 for the notion of a random closed set.

Lemma 2.1. For any $t \geq 0, S_{t}$ and $Z_{t}$ are random closed sets.

Proof. To prove the first assertion it suffices to show that $\left\{S_{t} \cap G=\emptyset\right\}$ is measurable for any open $G \subset \mathbb{R}^{d}$; see [14, Lemma 2.1.1]. Since $X$ is right continuous with left limits, it is clear that $S_{t} \cap G=\emptyset$ if and only if $X(u) \notin G$ for all rational numbers $u \leq t$. The second assertion is implied by [14, Thms. 12.3.5, 12.3.2].

Lemma 2.1 implies that $V\left(B[d-1], Z_{t}[1]\right)$ and $V_{d}\left(S_{t}+B\right)$ are random variables; see e.g. [13, p. 275] and [14, Thms. 12.3.5, 12.3.6].

Proof of Theorem 1.1. By [13, Eq. (5.1.18) and p. 277],

$$
V(B[d-1], K[1])=\frac{1}{d} \int_{S^{d-1}} h(K, u) S_{d-1}(B, d u)
$$

for all convex bodies $B, K \subset \mathbb{R}^{d}$, where $S_{d-1}(B, \cdot)$ is the surface area measure of $B$; see [13, Sec. 4.2]. Fubini's theorem and (2.1) yield that

$$
\mathbb{E} V(B[d-1], Z[1])=\frac{1}{d} \int_{S^{d-1}} \mathbb{E} h(Z, u) S_{d-1}(B, d u) .
$$

For any $u \in S^{d-1}$,

$$
\mathbb{E} h(Z, u)=\mathbb{E} \sup \left\{\langle x, u\rangle: x \in Z_{1}\right\}=\mathbb{E} \sup \{\langle X(s), u\rangle: s \in[0,1]\} .
$$


It follows from the definition of $\alpha$-stability that the one-dimensional Lévy process $Y=\langle X, u\rangle$ is symmetric $\alpha$-stable. By [1, Thm. 4a], $\sup \{Y(s): s \in[0,1]\}$ has a finite expectation. Differentiating equation (7b) in [1] (Spitzer's identity in continuous time), one can easily show that

$$
\mathbb{E} \sup \{Y(s): s \in[0,1]\}=\alpha \mathbb{E} Y(1)^{+}=\alpha \mathbb{E}(\langle u, X(1)\rangle)^{+},
$$

where $a^{+}:=\max \{0, a\}$ denotes the positive part of a real number $a$. It is shown in [10, Sec. 6.4] that $\mathbb{E}(\langle u, X(1)\rangle)^{+}=h(M, u)$ is the support function of a scaled variant of the associated zonoid $K$, namely

$$
M=\frac{1}{\pi} \Gamma\left(1-\frac{1}{\alpha}\right) K .
$$

Together with (2.1), this yields assertion (1.8).

Proof of Corollary 1.4. Since $K=B^{d} / \sqrt{2}$, Theorem 1.1] and (1.7) give

$$
\begin{aligned}
\mathbb{E} V_{1}(Z) & =\mathbb{E} \frac{d}{\kappa_{d-1}} V\left(B^{d}[d-1], Z[1]\right) \\
& =\frac{d}{\kappa_{d-1}} \frac{2}{\pi} \Gamma\left(1-\frac{1}{2}\right) V\left(B^{d}[d-1], \frac{B^{d}}{\sqrt{2}}[1]\right) \\
& =\sqrt{\frac{2}{\pi}} \frac{d \kappa_{d}}{\kappa_{d-1}}
\end{aligned}
$$

where we have used the fact that $V\left(B^{d}[d-1], B^{d}[1]\right)=V_{d}\left(B^{d}\right)$. The well-known formula $\kappa_{d}=\pi^{d / 2} / \Gamma(d / 2+1)$ and the obvious identity $d / \Gamma(d / 2+1)=2 / \Gamma(d / 2)$ yield the result.

Proof of Lemma 1.5. Without loss of generality assume that

$$
K=\left\{\left(x_{1}, \ldots, x_{d}\right) \in \mathbb{R}^{d}:\left(\frac{x_{1}}{c_{1}}\right)^{2}+\cdots+\left(\frac{x_{d}}{c_{d}}\right)^{2} \leq 1\right\} .
$$

Consider the linear transform $x \mapsto f(x)=\left(c_{1}^{-1} x_{1}, \ldots, c_{d}^{-1} x_{d}\right)$. Then $f(K)=B^{d}$, while the affine equivariance of the mixed volumes and the fact that the determinant of $f$ is $\left(c_{1} \cdots c_{d}\right)^{-1}$ yield that

$$
\begin{aligned}
V_{1}(K) & =\frac{d}{\kappa_{d-1}} V\left(B^{d}[d-1], K[1]\right) \\
& =\frac{d c_{1} \cdots c_{d}}{\kappa_{d-1}} V\left(f\left(B^{d}\right)[d-1], f(K)[1]\right) \\
& =\frac{d c_{1} \cdots c_{d}}{\kappa_{d-1}} V\left(B^{d}[1], F[d-1]\right) \\
& =\frac{d c_{1} \cdots c_{d}}{\kappa_{d-1}} \frac{\kappa_{1}}{d} V_{d-1}(F),
\end{aligned}
$$

where $F=f\left(B^{d}\right)$ is the ellipsoid with semi-axes $c_{1}^{-1}, \ldots, c_{d}^{-1}$, namely the polar body to $K$. It remains to note that $\kappa_{1}=2$. 
Proof of Proposition [1.6. Kubota's formula (see e.g. [13, Eq. (5.3.27)]) yields

$$
V_{2}(Z)=\frac{d(d-1) \kappa_{d}}{2 \kappa_{2} \kappa_{d-2}} \int_{G_{2}} V_{2}(Z \mid L) \nu_{2}(d L)
$$

where $G_{2}$ denotes the set of all 2-dimensional linear subspaces of $\mathbb{R}^{d}, \nu_{2}$ is the Haar measure on $G_{2}$ with $\nu_{2}\left(G_{2}\right)=1$ and $Z \mid L$ denotes the image of $Z$ under the orthogonal projection onto the linear subspace $L$. By Fubini's theorem,

$$
\mathbb{E} V_{2}(Z)=\frac{d(d-1) \kappa_{d}}{2 \kappa_{2} \kappa_{d-2}} \int_{G_{2}} \mathbb{E} V_{2}(Z \mid L) \nu_{2}(d L) .
$$

The spherical symmetry of Brownian motion implies that $\mathbb{E} V_{2}(Z \mid L)$ does not depend on $L$. Assume that $L=\left\{\left(x_{1}, x_{2}, 0, \ldots, 0\right): x_{1}, x_{2} \in \mathbb{R}\right\}$. Now it is clear from the definition of the $d$-dimensional Brownian motion that the random closed set $Z \mid L$ is the convex hull of a Brownian path in L. By Remark (a) in [3, p. 149] (see also [8]) we have $\mathbb{E} V_{2}(Z \mid L)=\pi / 2$. Therefore,

$$
\mathbb{E} V_{2}(Z)=\frac{d(d-1) \kappa_{d}}{2 \kappa_{2} \kappa_{d-2}} \frac{\pi}{2},
$$

and the result follows by a straightforward calculation.

Proof of Theorem 1.7. Note that a simple rescaling argument makes it possible to assume that $K=B_{\alpha^{\prime}}^{d}$ is the unit $\ell_{\alpha^{\prime}}$-ball. By self-similarity and the dominated convergence theorem we have

$$
\begin{aligned}
\lim _{t \rightarrow 0} t^{-1 / \alpha}\left(\mathbb{E} V_{d}\left(S_{t}+B\right)-V_{d}(B)\right) & =\lim _{t \rightarrow 0} t^{-1 / \alpha}\left(\mathbb{E} V_{d}\left(t^{1 / \alpha} S_{1}+B\right)-V_{d}(B)\right) \\
& =\lim _{t \rightarrow 0} t^{-1}\left(\mathbb{E} V_{d}\left(t S_{1}+B\right)-V_{d}(B)\right) \\
& =\mathbb{E} \lim _{t \rightarrow 0} t^{-1}\left(V_{d}\left(t S_{1}+B\right)-V_{d}(B)\right) .
\end{aligned}
$$

In order to justify the application of the dominated convergence theorem, define

$$
\begin{aligned}
& Y_{j}=\sup \left\{X_{j}(s): s \in[0,1]\right\}, \\
& \tilde{Y}_{j}=\inf \left\{X_{j}(s): s \in[0,1]\right\}, \quad j=1, \ldots, d .
\end{aligned}
$$

As noted in the proof of Theorem 1.1, $Y_{j}$ has a finite expectation. Since $-\tilde{Y}_{j}$ has the same distribution as $Y_{j}, \tilde{Y}_{j}$ also has a finite expectation. From (1.6) we obtain for all $t \in(0,1]$ that

$$
\begin{aligned}
t^{-1} V_{d}\left(t S_{1}+B\right)-V_{d}(B) & \leq t^{-1}\left(V_{d}(t Z+B)-V_{d}(B)\right) \\
& =\sum_{j=0}^{d-1}\left(\begin{array}{l}
d \\
j
\end{array}\right) t^{d-j-1} V(B[j], Z[d-j]) \\
& \leq \sum_{j=0}^{d}\left(\begin{array}{l}
d \\
j
\end{array}\right) V(B[j], Z[d-j]) \\
& =V_{d}(Z+B) .
\end{aligned}
$$


Furthermore,

$$
Z+B \subset \underset{j=1}{\stackrel{d}{\times}}\left[\tilde{Y}_{j}-h_{B}\left(-e_{j}\right), Y_{j}+h_{B}\left(e_{j}\right)\right],
$$

where $e_{j}$ denotes the $j$ th unit vector. Thus

$$
t^{-1}\left(V_{d}\left(t S_{1}+B\right)-V_{d}(B)\right) \leq \prod_{j=1}^{d}\left(Y_{j}+h_{B}\left(e_{j}\right)-\tilde{Y}_{j}+h_{B}\left(-e_{j}\right)\right)
$$

for $t \in(0,1]$. This is a product of integrable independent random variables and hence has finite expected value.

By [7, Cor. 3.2(2)],

$$
\lim _{t \rightarrow 0} t^{-1}\left(V_{d}\left(t S_{1}+B\right)-V_{d}(B)\right)=\int_{S^{d-1}} h(Z, u) S_{d-1}(B, d u)
$$

and using Theorem 1.1 and (2.1) we conclude from (2.2) that

$$
\begin{aligned}
\lim _{t \rightarrow 0} t^{-1 / \alpha} \mathbb{E}\left(V_{d}\left(S_{t}+B\right)-V_{d}(B)\right) & =\mathbb{E} \int_{S^{d-1}} h(Z, u) S_{d-1}(B, d u) \\
& =d \mathbb{E} V(B[d-1], Z[1]) \\
& =d \frac{\alpha}{\pi} \Gamma\left(1-\frac{1}{\alpha}\right) V(B[d-1], K[1]) .
\end{aligned}
$$

\section{REFERENCES}

1. N. H. Bingham, Maxima of sums of random variables and suprema of stable processes, Z. Wahrsch. verw. Gebiete 26 (1973), 273-296. MR0415780 (54:3859)

2. F. Bowman, Introduction to elliptic functions with applications, Dover Publ., New York, 1961. MR0132214 (24:A2060)

3. M. Cranston, P. Hsu, and P. March, Smoothness of the convex hull of planar Brownian motion, Ann. Probab. 17 (1989), 144-150. MR972777 (89m:60190)

4. W. J. Firey, Some means of convex bodies, Trans. Amer. Math. Soc. 129 (1967), 181-217. MR0234349 (38:2666)

5. R. Getoor, Some asymptotic formula involving capacity, Z. Wahrsch. verw. Gebiete 4 (1965), 248-252. MR0190988 (32:8397)

6. O. Kallenberg, Foundations of modern probability, 2nd ed., Springer, New York, 2002. MR.1876169 (2002m:60002)

7. M. Kiderlen and J. Rataj, On infinitesimal increase of volumes of morphological transforms, Mathematika 53 (2006), 103-128. MR2304055 (2008g:60034)

8. S. N. Majumdar, A. Comtet, and J. Randon-Furling, Random convex hulls and extreme value statistics, J. Stat. Phys. 138 (2010), 955-1009. MR2601420 (2011c:62166)

9. I. Molchanov, Theory of random sets, Springer, London, 2005. MR2132405 (2006b:60004)

10. __ Convex and star shaped sets associated with multivariate stable distributions. I. Moments and densities, J. Multivariate Anal. 100 (2009), 2195-2213. MR2560363(2011g:60033)

11. J. Rosen, The asymptotics of stable sausages in the plane, Ann. Probab. 20 (1992), 29-60. MR.1143411 (93a:60063)

12. G. Samorodnitsky and M. S. Taqqu, Stable non-Gaussian random processes, Chapman \& Hall, New York, 1994. MR.1280932 (95f:60024)

13. R. Schneider, Convex bodies. The Brunn-Minkowski theory, Cambridge University Press, Cambridge, 1993. MR.1216521 (94d:52007)

14. R. Schneider and W. Weil, Stochastic and integral geometry, Springer, Berlin, 2008. MR 2455326 (2010g:60002)

15. F. Spitzer, Electrostatic capacity, heat flow, and Brownian motion, Z. Wahrsch. verw. Gebiete 3 (1964), 110-121. MR 0172343 (30:2562) 
16. L. Takacs, Expected perimeter length, Amer. Math. Monthly 87 (1980), 142. MR1539300

17. G. Tee, Surface area and capacity of ellipsoids in $n$ dimensions, New Zealand J. Math. 34 (2005), 165-198. MR2195834(2006i:49060)

AG Statistik, TU Kaiserslautern, 67653 Kaiserslautern, Germany

E-mail address: kampf@mathematik.uni-kl.de

Institut für Stochastik, Karlsruhe Institute of Technology, 76128 Karlsruhe, GerMANY

E-mail address: guenter.last@kit.edu

Institute of Mathematical Statistics and Actuarial Science, University of Bern, Sidlerstrasse 5, 3012 Bern, Switzerland

E-mail address: ilya.molchanov@stat.unibe.ch 